\title{
BACTERIAL STI
}

\section{The prevalence of proline iminopeptidase negative Neisseria gonorrhoeae throughout England and Wales}

\author{
S Alexander, I M C Martin, K Fenton, C A Ison
}

Sex Transm Infect 2006;82:280-282. doi: 10.1136/sti.2005.018424

\begin{abstract}
See end of article for authors' affiliations ......................

Correspondence to: Dr Sarah Alexander Sexually Transmitted Bacteria Reference Laboratory, Health Protection Agency, Centre For Infections, Colindale Avenue, London NW9 $5 \mathrm{HT}$, UK; sarah. alexander@hpa.org.uk
\end{abstract}

Accepted for publication 8 March 2006 ntification of Neisseria gonorrhoeae is an essential element of Background: The accurate laboratory identification of Neisseria gonorrhoeae is an essential element of
the diagnosis of gonorrhoea and is particularly important for medicolegal cases. The detection of proline iminopeptidase (Pip) activity is widely used as a marker for gonococci, although Pip negative $N$ gonorrhoeae isolates have been shown to generate false negative identifications when using biochemical kits. This study aimed to determine the frequency of Pip negative gonococci in England and Wales.

Methods: A total of 2055 isolates were collected from consecutive patients attending 26 genitourinary medicine centres as part of the Gonococcal Resistance to Antimicrobials Surveillance Programme (GRASP). Upon receipt the identity of all isolates was confirmed using N gonorrhoeae specific monoclonal antibodies and the Pip status was determined using the Gonochek II kit.

Results: The overall prevalence of Pip negative isolates was found to be $4.33 \%$. Significant geographical variation was observed between isolates from centres outside London $(p \leqslant 0.001)$. Variation was also observed within London between the nine different clinics submitting isolates $(p=0.025)$. There was also a higher frequency of these isolates among men who have sex with men $(p \leqslant 0.001)$, which may account for geographical variations.

Conclusion: Pip negative $N$ gonorrhoeae isolates are a very serious cause for concern as currently all biochemical test kits available within the United Kingdom require the presence of the Pip enzyme for an unambiguous identification of this pathogen. Raising awareness of the current prevalence of Pip negative $\mathrm{N}$ gonorrhoeae isolates is critical for the successful control of gonorrhoea.
G onorrhoea is the second most common bacterial sexually transmitted infection (STI) in the United Kingdom and control of this disease is dependent on the successful, rapid, and accurate identification of the pathogen Neiserria gonorrhoeae. One of the most common methods routinely used in clinical microbiology settings for the identification of this pathogen, is the detection of preformed enzymes. The use of preformed enzymes as a tool for the differentiation of Neisseria spp was first described by D'Amato et al, who reported the production of the enzymes gamma glutamyl transferase (GGT) by $N$ meningitidis and proline iminopeptidase (Pip) by $N$ gonorrhoeae. ${ }^{1}$ Following this, several commercial kits were developed which exploit the presence of these enzymes for the purposes of discriminating between $N$ gonorrhoeae and other Gram negative diplococci.

Molecular cloning and characterisation of the $N$ gonorrhoeae pip gene was performed in 1993 where it was confirmed that pip was a single copy gene and, while non-essential for growth in vitro was present in virtually all isolates of $N$ gonorrhoeae. ${ }^{2}$ However since this time the widespread distribution of Pip negative $N$ gonorrhoeae throughout England has been reported. ${ }^{3}$ Currently all biochemical kits available within the United Kingdom for the identification of Neisseria spp, including the Neisseria preformed enzyme test (PET), Gonochek II, RapID NH, and the API NH monitor the presence of this enzyme. Consequently Pip negative gonococcal isolates generate ambiguous identifications ranging from "doubtful $N$ gonorrhoeae" to "presumptive Kingella kingea" when examined using these kits. ${ }^{4}$ This problem becomes more significant when using test kits such as the Neisseria PET test and the Gonochek II where a presumptive $N$ gonorrhoeae identification is based solely on the presence of this enzyme. False positive $N$ gonorrhoeae identifications can also be generated when using these kits as many non-pathogenic Neisseria spp are Pip positive and some have been reported to grow well on selective media. ${ }^{45}$ However despite these reports the use of preformed enzyme based tests kits remains popular and they are widely used for the routine identification of $N$ gonorrhoeae.

While it is acknowledged that commercially available biochemical kits generate ambiguous results when used to examine isolates of $N$ gonorrhoeae which lack Pip, the incidence of isolates lacking this enzyme is currently unknown. This study aimed to determine the frequency of Pip negative $N$ gonorrhoeae within a representative sample from surveillance isolates in England and Wales.

\section{METHODS}

A total of 2055 isolates of $N$ gonorrhoeae from consecutive patients attending 26 genitourinary medicine (GUM) clinics throughout England and Wales collected over a 3 month period during 2004 as part of the Gonococcal Resistance to Antimicrobials Surveillance Programme (GRASP) were tested. All isolates were positively identified as $N$ gonorrhoeae at the local laboratory using a range of methods and then forwarded to the Sexually Transmitted Bacterial Reference Laboratory for testing. Upon receipt, isolates were subcultured on GC agar base (Beckton Dickinson) supplemented with Vitox and purity was determined by a Gram stain. The identity of all isolates was further confirmed using in-house monoclonal antibodies specific to $N$ gonorrhoeae. In a few instances where in house monoclonal antibodies failed to react, identity was confirmed using the API NH kit

Abbreviations: GGT, gamma glutamyl transferase; GRASP, Gonococcal Resistance to Antimicrobials Susceptibility Programme; GUM, genitourinary medicine; MSM, men who have sex with men; PET, preformed enzyme test; Pip, proline iminopeptidase; STI, sexually transmitted infections 
Table 1 The proline iminopeptidase (Pip) status of Neisseria gonorrhoeae isolates, broken down by submitting centres

\begin{tabular}{ll}
\hline & $\begin{array}{l}\text { Frequency of Pip } \\
\text { negative N gonorrhoeae } \\
\text { isolates (no of Pip } \\
\text { negative isolates) }\end{array}$ \\
Hospital (no of isolates tested) & $\mathbf{4 . 2 7 ( 4 6 )}$ \\
\hline Total Outer London (1078) & $0.00(0)$ \\
New Cross Hospital, Wolverhampton (29) & $0.00(0)$ \\
Gloucestershire Royal Hospital (19) & $1.98(2)$ \\
Northern General Hospital Sheffield (101) & $0.00(0)$ \\
Newcastle (35) & $5.36(6)$ \\
Manchester Royal Infirmary (112) & $1.94(2)$ \\
Royal Liverpool Hospital (103) & $2.80(3)$ \\
Birmingham Queen Elizabeth Hospital (107) & $2.97(3)$ \\
Nottingham City Hospital (101) & $0.00(0)$ \\
The Royal Berkshire Hospital, Reading (37) & $21.43(3)$ \\
Addenbrooke's Hospital, Cambridge (14) & $10.08(13)$ \\
Bristol Royal Infirmary (129) & $0.00(0)$ \\
Luton and Dunstable Hospital (33) & $0.00(0)$ \\
Cardiff Royal Infirmary (72) & $17.86(10)$ \\
The Royal Sussex County Hospital Brighton & \\
(56) & $4.65(4)$ \\
Leeds General Infirmary (86) & $0.00(0)$ \\
The Royal Gwent Hospital, Newport (25) & $0.00(0)$ \\
Northampton General Hospital (19) & $4.40(43)$ \\
Total London (977) & $2.51(6)$ \\
Kings College Hospital (239) & $2.32(4)$ \\
Homerton University Hospital (176) & $9.29(13)$ \\
University College London Hospital (140) & $6.20(8)$ \\
St Mary's Hospital (129) & $3.13(3)$ \\
St George's Hospital (96) & $8.33(5)$ \\
Chelsea and Westminster healthcare NHS & \\
Trust, Victoria Clinic (60) & $5.08(3)$ \\
Chelsea and Westminster, The John Hunter & \\
Clinic (59) & \\
Charing Cross Hospital (43) & $0.00(0)$ \\
Queen Elizabeth Hospital Woolwich (35) & 2.86 (1) \\
\hline
\end{tabular}

(bioMerieux). Following identification, the Pip status of all isolates was established using the Gonochek II test (E-Y Laboratories Inc).

Bivariate analysis using the $\chi^{2}$ test was applied to the whole data set where possible. Extended patient information such as sexual orientation was only available for isolates of $N$ gonorrhoeae from patients attending GUM clinics. Therefore isolates originating from patients attending GP practices or other hospital departments were not included in some of the final analysis.

\section{RESULTS}

The overall prevalence of Pip negative $N$ gonorrhoeae was found to be $4.33 \%$ ( 89 of 2055). There was geographical variation observed in the frequency of Pip negative $N$ gonorrhoeae isolates submitted from centres outside London $(\mathrm{p} \leqslant 0.001$ : table 1$)$. Variation in prevalence was also

Table 2 The relation between the frequency of proline iminopeptidase negative $\mathrm{N}$ gonorrhoeae and gender and/or sexual orientation of patient $(p \leqslant 0.001)$.

\begin{tabular}{ll}
\hline & $\begin{array}{l}\text { Frequency of Pip negative } \\
\text { N gonorrhoeae isolates (no of Pip } \\
\text { negative isolates) }\end{array}$ \\
\hline MSM (460) & $8.47(39)$ \\
Male (575) & $2.43(14)$ \\
Female (541) & $2.40(13)$ \\
\hline
\end{tabular}

Total $=1576$, excludes 479 isolates where the sexual orientation and/or gender of the patient was unknown. observed within London, between the nine different clinics submitting isolates $(\mathrm{p}=0.025$ : table 1$)$.

The reason for the difference in the prevalence of Pip negative isolates throughout different cities in England and Wales and even between different clinics within London is unknown. Differences in geographical prevalence can not be explained by some of the primary sites using only preformed enzyme kits (for example, Gonochek or the Neisseria PET Test) for $N$ gonorrhoeae identification, which would lead to an under-representation of Pip negative isolates. All clinics or centres submitting isolates used either adequate or multiple methods for the identification of all gonococcal isolates. ${ }^{6}$ There was however a clear association between prevalence and sexual orientation (table 2), with a higher prevalence of Pip negative isolates being significantly associated with infection in men who have sex with men (MSM) $(p \leqslant 0.001)$. As expected the number of isolates sourced from MSM submitted from the 26 different centres was not equal and this is clearly a reflection of the MSM population being unevenly distributed throughout England and Wales, with centres such as Brighton and Manchester contributing the highest number of isolates sourced from this patient group.

\section{DISCUSSION}

This study has highlighted that $4.33 \%$ of $N$ gonorrhoeae isolated in England and Wales lack the Pip enzyme, using a representative collection of isolates from a sentinel surveillance programme. This is a significant problem given that there are several kits, which monitor solely the presence of this enzyme for the detection of this pathogen. Pip negative $N$ gonorrhoeae isolates have previously been shown to generate a false negative identification using biochemical tests. ${ }^{47}$ However confusion over which kits detect the presence of this enzyme may be attributed to incorrect terminology used in the manufacturers' kit instructions. The enzyme proline iminopeptidase is a hydrolase, which selectively removes the $\mathrm{N}$-terminal proline residue from peptides. However names used for this enzyme in biochemical kit inserts include proline aminopeptidase, prolylaminopeptidase, and proline arylamdase. It is the responsibility of manufactures to adopt consistent, up to date, and scientifically correct names for the enzymes that their kits are monitoring. This would ensure that users of particular tests are able to make informed judgments as to the limitations of particular test systems in response to published literature.

Until relatively recently Pip negative isolates of $N$ gonorrhoeae had not been widely reported. This is in contrast with $N$ lactamica and $N$ meningitidis where both Pip negative and Pip positive isolates have both been documented. ${ }^{8}{ }^{9}$ In a method evaluation carried out in 1991, only two out of 398 isolates of $N$ gonorrhoeae were found to be Pip negative. ${ }^{10}$ Interestingly a report from the Gonococcal Reference Unit in Bristol during 2001, raised awareness of this as 17 Pip negative $N$ gonorrhoeae isolates had been forwarded to them over a 5 week period for confirmatory identification. ${ }^{3}$ The emergence and apparent increase in the prevalence of these isolates suggests that they may have a selective advantage. It is possible to speculate that such isolates are more likely to be misidentified and therefore not treated. Alternatively it is also plausible that the mutation which renders the Pip enzyme inactive does not have any effect on the fitness of the gonococcal isolate and that the only reason for their apparent increase in prevalence and widespread geographical dissemination could be that they have found a host in a highly sexually active community. Alternatively this apparent increase in prevalence could just be an artefact of increasing awareness of Pip negative gonococcal isolates, with laboratories which have previously been using inadequate methods for gonococcal identification 


\section{Key messages}

- Proline iminopeptidase (Pip) negative Neisseria gonorrhoeae isolates generate anomalous results when examined by commercially available biochemical test kits

- The overall prevalence of Pip negative $\mathrm{N}$ gonorrhoeae isolates in England and Wales is 4.33\%

- There is a higher prevalence of Pip negative $N$ gonorrhoeae in men who have sex with men

having adopted the national standard guidelines which has resulted in an increase in reporting. ${ }^{6}$

This study suggests that all clinical isolates of $N$ gonorrhoeae be identified using two methods, preferably both biochemical and immunological, especially if the biochemical method relies totally on the detection of preformed enzymes. ${ }^{6}$ This is of particular importance when testing isolates taken from children and victims of sexual abuse where medicolegal issues must be addressed and in these cases the use of carbohydrate tests is particularly recommended.

\section{ACKNOWLEDGEMENTS}

We thank Caroline Murphy, Hemanti Patel, Mariam Perault, and Elizabeth Rudd for assistance in the testing of isolates and coordinating the data; and TSC BioSciences for the provision of Gonochek II kits at a reduced price.

We also thank colleagues at the following collecting centres participating in GRASP for the provision of $N$ gonorrhoeae isolates: Cambridge $-M$ Farrington, C Carne (Addenbrooke's NHS Trust); Luton-R Mulla (Luton Microbiology Laboratory), T Balachandran (Luton and Dunstable Hospital); London-B Azadian (Chelsea and Westminster Hospital), K McLean (Charing Cross Hospital), A McOwan (Victoria Clinic), F Boag (John Hunter Clinic), A Karcher, M Nathan (Homerton Hospital), M Graver, M Tenant-Flowers (King's Healthcare NHS Trust), P Hay, R Holliman, (St George's Hospital), A Jepson, L Green (St Mary's Hospital), S Morris Jones, A Robinson (University College London Hospitals), S Bragman, J Russell (Queen Elizabeth Hospital NHS Trust); Liverpool—T Neal, P Carey (Royal Liverpool Hospital); Manchester-A Qamruddin, D Mandal (Manchester Royal Infirmary); Leeds-D Gascoyne-Binizi, M Waugh (Leeds General Infirmary); Newcastle-J Magee, N Sankar (Newcastle General Hospital); Brighton-J Paul, D Williams (Royal Sussex County Hospital); Northampton-M Minassian, L Riddell (Northampton General Hospital); Reading-A Stacey, G Wildman, (Royal Berkshire Hospital); Bristol-R Spencer, $\mathrm{P}$ Horner (Bristol Royal Infirmary); Gloucester-M Logan, $M$ Sulaiman (Gloucestershire Royal Hospital); Nottingham-T Boswell
(University Hospital Queens Medical Centre), C Bignell (Nottingham City Hospital); Sheffield-P Zadik (Northern General Hospital), G Kinghorn (Royal Hallamshire Hospital); Cardiff-I Hosein (University Hospital of Wales), H Birley (Cardiff Royal Infirmary); Newport-E Kubiak, R Das (Royal Gwent Hospital); BirminghamM Gill (University Hospital Birmingham), J Ross (Whittall Street Clinic); Wolverhampton-J Fernando, H Jones (New Cross Hospital).

\section{CONTRIBUTORS}

SA was responsible for the isolate screening methods and data analysis; IM was responsible for supervision on the practical stage of the project; KF designed the isolate and patient data, collection protocol and was GRASP lead investigator; CI was responsible for initiation and supervision of the project. All authors have read and approved the final version of this manuscript.

\section{Authors' affiliations}

S Alexander, I M C Martin, C A Ison, Sexually Transmitted Bacteria Reference Laboratory, Health Protection Agency, Centre For Infections, Colindale Avenue, London NW9 5EQ, UK

K Fenton, Centers for Disease Control and Prevention, 1600 Clifton Road, Atlanta, GA 30333, USA

Conflict of interest: none.

\section{REFERENCES}

1 D'mato RF, Erique LA, Tomfohrde KM, et al. Rapid identification of Neisseria gonorrhoeae and Neisseria meningtidis by using enzymatic profiles. J Clin Microbiol 1978;77:77-81.

2 Albertson NH, Koomey M. Molecular cloning and characterization of a proline iminopeptidase gene from Neisseria gonorrhoeae. Mol Microbiol 1993;9:1203-11

3 Health Protection Agency. Prolyliminopeptidase-negative isolates of Neisseria gonorrhoeae. Commun Dis Rep CDR Wkly [serial online] 11 (49). 2001. Available at http://www.hpa.org.uk/cdr/archives/2001/cdr4901.pdf.

4 Alexander S, Ison C. An evaluation of commercial kits for the identification of Neisseria gonorrhoeae. J Med Microbiol 2005;54:827-31.

5 Knapp JS, Totten PA, Mulks MH, et al. Characterisation of Neisseria cinerea, a non-pathogenic species isolated on Martin-Lewis Medium selective for pathogenic Neisseria spp. J Clin Microbiol 1984;19:63-7.

6 Health Protection Agency. Identification of Neisseria species. National Standard Method. 2005. Available at http://www.hpastandardmethods.org.uk/documents/bsopid/pdf/bsopid6.pdf.

7 Blackmore T, Hererra G, Shi S, et al. Characterization of prolyl iminopeptidase-deficient Neisseria gonorrhoeae. J Clin Microbiol 2005:43:4189-90.

8 Sperry JF, Cohenford MA, Campognone P, et al. Increased detection of prolylaminopeptidase in Neisseria meningitidis by Identicult-Neisseria. J Clin Microbiol 1986;24:145-5.

9 Yajiko DM, Chu A, Hadley WK. Rapid confirmatory identification of $\mathrm{N}$ gonorrhoeae with lectins and chromogenic substrates. J Clin Microbiol 1984; 19:145.

10 Dealler SF, Gough KR, Campbell L, et al. Identification of Neisseria gonorrhoeae using the Neisstrip rapid enzyme detection test. J Clin Path 1991;44:376-9. 